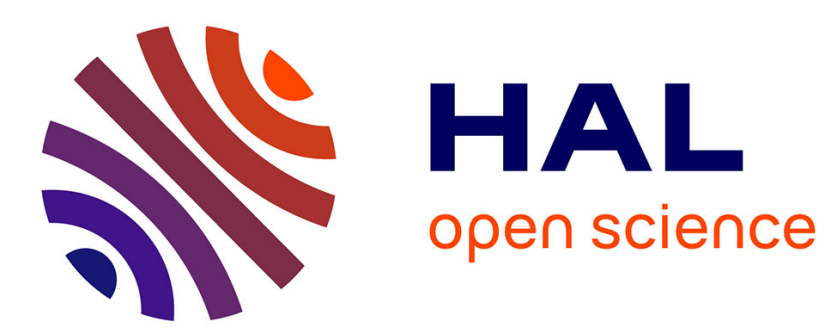

\title{
Minorités sexuelles et rapport au corps : une recherche quantitative
}

Arnaud Alessandrin, Marielle Toulze

\section{To cite this version:}

Arnaud Alessandrin, Marielle Toulze. Minorités sexuelles et rapport au corps: une recherche quantitative. Santé Publique, 2020. hal-02460887

\section{HAL Id: hal-02460887 \\ https://hal.science/hal-02460887}

Submitted on 30 Jan 2020

HAL is a multi-disciplinary open access archive for the deposit and dissemination of scientific research documents, whether they are published or not. The documents may come from teaching and research institutions in France or abroad, or from public or private research centers.
L'archive ouverte pluridisciplinaire HAL, est destinée au dépôt et à la diffusion de documents scientifiques de niveau recherche, publiés ou non, émanant des établissements d'enseignement et de recherche français ou étrangers, des laboratoires publics ou privés. 


\section{Minorités sexuelles ${ }^{1}$ et rapport au corps : une recherche quantitative ${ }^{2}$ Arnaud Alessandrin, Marielle Toulze}

Pour citer l'article :

Arnaud Alessandrin, Marielle Toulze, "Minorités sexuelles et rapport au corps : une recherche quantitative », Santé publique $31 / N^{\circ} 5,557-561,2019$

Résumé

Introduction: Si les questions liées à la santé sexuelle et reproductive des gays et des lesbiennes sont bien renseignées, d'autres, relatives à la santé globale et au rapport au corps, sont moins souvent abordées.

But de l'étude: Issu de la recherche "Santé LGBTI », cet article tente de combler ce manque en se donnant pour objectif de restituer les résultats d'une enquête quantitative réalisée en France en 2017 en se focalisant plus précisément sur les personnes gays et lesbiennes. Aussi, les résultats de cette recherche sont mis en regard avec la littérature disponible sur cette question.

Résultats: Par la mesure d'indices comme le recours aux régimes, le souci du poids ou la pratique sportive, nous tendons à montrer que les personnes homosexuelles n'ont pas le même rapport au corps que les personnes hétérosexuelles et qu'entre les lesbiennes et les gays des différences apparaissent. Conclusions: Si les résultats de cette étude sont

\footnotetext{
${ }^{1}$ Les « minorités sexuelles » font référence l'ensemble des personnes non hétérosexuelles. Quant à la notion de " minorité de genre », elle renvoie aux personnes qui changent de sexe ou de change.

2 Cette recherche est financée par la Délégation interministérielle à la lutte contre le racisme, l'antisémitisme et la haine anti-LGBT (Dilcrah) et portée par l'Association de recherche et d'étude sur la santé, la ville et les inégalités (Aresvi) (2017-2018). S'ajoutent aux auteures du présent article: Meidani Anastasia, maîtresse de conférence en sociologie- et chercheuse associée au laboratoire interdisciplinaire "Solidarités, Sociétés, Territoires » (Lisst) (Université Jean-Jaurès, Toulouse), Johanna Dagorn, sociologue et chercheuse associée au -laboratoire "Cultures, Éducation, Sociétés » (Laces) (Université de Bordeaux), Gabrielle Richard, Sociologue et chercheuse associée au Laboratoire interdisciplinaire de recherche sur les transformations des pratiques éducatives et des pratiques sociales (Lirtes) (Université Paris Est-Creteil) et Marouchka Dubot, masterante à I'université de Toulouse Jean-Jaurès et Clément Reverse, doctorant au Centre Émile-Durkheim (Université de Bordeaux). L'ensemble des résultats de cette recherche est publié dans Alessandrin A, Dagorn J, Meidani A, Richard G, Toulze M. Santé LGBT. Bord de l'eau ; 2020.
} 
concomitants avec les données internationales en la matière, ils dessinent aussi des angles morts en termes de rapport au corps des sous-cultures homosexuelles.

Mots-clés : Sexualité ; Genre ; Corps.

Introduction

Le rapport à la santé des personnes lesbiennes, gays, bisexuel.le.s, transgenres, intersexes et queer (LGBTIQ) se mesure souvent à l'aune des questions de santé sexuelles et reproductives en ce qui concerne les minorités sexuelles, et des questions de santé mentale en ce qui concerne les minorités de genre1. Pourtant, d'autres problématiques traversent les parcours de santé des populations LGBTIQ, par exemple la question de leur santé globale en lien avec les médecins généraux [1], celle du vieillissement [2] ou bien la question de la santé scolaire $[3,4]$. Mais au-delà de pathologies ciblées, une question semble rester en suspens dans la recherche francophone, notamment: celle du rapport au corps des gays et des lesbiennes, qu'il s'agisse de prise de poids, de musculature ou de minceur. D'ailleurs, les travaux sur ces thématiques, même lorsqu'ils abordent les questions du sex-ratio face aux enjeux épidémiologiques de l'obésité ou du surpoids, évincent les notions d'identité sexuelle ou de genre [5]. Si la focale mise sur le virus de l'immunodéficience humaine (VIH) pourrait expliquer la mise sous silence d'autres problématiques de santé, l'explosion récente des études gays et lesbiennes (gay and lesbiennes studies) interroge la rareté de recherches relatives à la santé des LGBTIQ en France $[6,7]$. C'est pourquoi, à travers une recherche quantitative, nous souhaitons interroger la manière dont les gays et les lesbiennes vivent leurs corps. II ne s'agit donc pas tant pour nous de vérifier les indices de masse corporelle (IMC) des personnes interrogées, notamment parce que cette mesure est largement discutée [8], mais bel et bien de travailler les perceptions corporelles [9] à travers des indices comme l'activité physique, la perte de poids, le régime ou le surpoids.

Santé LGBTI : retour sur une méthode 
De juin 2017 à septembre de la même année, une équipe de la recherche pluridisciplinaire "Santé LGBTI »2 a diffusé un questionnaire portant sur la santé et le rapport au corps méthodologie, nous avons choisi de suivre deux méthodes concomitantes : une passation de questionnaire via internet et une synthèse des travaux faisant écho à nos interrogations. À travers les mots-clés comme "obésité », " poids ", " musculation », "régime » ou " corps », nous avons recueilli des données quantitatives ainsi que des analyses qui mettent en lien les études LGNT (LGBT-studies) et la sociologie du corps et de la santé. Nous discuterons ultérieurement ces résultats, mais soulignons d'ores et déjà que rares sont les écrits francophones qui abordent cette question, sinon très marginalement [10]. En effet, qu'il s'agisse de rapports institutionnels (comme le rapport Obépi de 2012 [11]) ou de recherches plus académiques $[12,13]$, la dimension genrée et sexuelle n'apparaît jamais. Ce constat engage donc la recherche dans une perspective comparative afin de mettre en lumière nos résultats.

Le questionnaire quant à lui a bénéficié d'une durée de diffusion de trois mois. Grâce aux réseaux associatifs ${ }^{3}$, à des pages institutionnelles ${ }^{4}$ et à des relais médiatiques communautaires ${ }^{5}$, ce questionnaire a atteint 1087 réponses complètes. II se composait de trois grands axes thématiques : la santé scolaire, la cancérologie [14] et des questions relatives au rapport aux corps. Sur ce dernier aspect, qui nous intéresse pour le présent article, trois sous-thèmes ont été abordés : le rapport à la perte de poids, le rapport à la prise de poids et celui du sport et de la musculature. Pour chacune de ces thématiques, une question ouverte et une question fermée ont été posées. Les répondantes et répondants se composent de $44 \%$ de personne s'identifiant comme «hommes » et autant comme «femmes », et $16 \%$ comme «trans», « intersexes» ou "non binaires». On compte également $62 \%$ de personnes homosexuelles, $21 \%$ de bisexuel.le.s, $4 \%$ d’hétérosexuelles et $13 \%$ se déclarant " pansexuel.le.s » ou » assexuel.le.s ». La réparti-tion par catégories socioprofessionnelles est la suivante : $26 \%$ d'employées, $20 \%$ de cadres, $24 \%$ d'inactifs et inac-tives (étudiant.e.s et retraité.e.s principalement), $12 \%$ de professions intermédiaires, $7 \%$ de professions libérales, $3 \%$ d'artisan.e.s/commerçant.e.s, $7 \%$ d'ouvrier.ère.s et $1 \%$ d'agricultrices et d'agriculteurs. Du côté de la réparti-tion par âge, 35 \% des répondant.e.s avaient moins de 25 ans lors de l'enquête, $45 \%$, entre 25 et 50 ans et $20 \%$, plus de 50 ans. Le mode de passation par internet peut expliquer les variations de répartition de populations par rapport à la moyenne nationale (peu d'ouvrier.ère.s et beaucoup d'étudiant.e.s, par exemple). En comparant les données internationales disponibles et celles issues de cette enquête, que pouvons-nous dire

\footnotetext{
${ }^{3}$ Nous remercions tout particulièrement les associations MAG-LGBT, I'Inter LGBT, le centre LGBT de Bordeaux et la Fédération nationale des centres LGBT.

${ }^{4}$ Principalement celle de la Direction interministérielle de lutte contre les discriminations.

${ }^{5}$ Les journaux Têtu et le site de rencontre Hornet-France ont partagé cette enquête avec leurs abonnés.
} 
? Autrement dit, y a-t-il des spécificités homosexuelles dans ce rapport au poids et, parallèlement, existe-t-il des différences entre les gays et les lesbiennes ?

Résultats

Si nous exploitons quelques grandes tendances de notre enquête, parmi les personnes sondées qui ne se sont « jamais » préoccupées de leur prise de poids (soit 190 personnes), on retrouve $53 \%$ de femmes, $12 \%$ de personnes trans (celles-ci n'ayant pas voulu répondre " homme » ou "femme ») et non binaires et $35 \%$ d'hommes. Les personnes s'identifiant comme "femmes" (quasi exclusivement des lesbiennes) semblent donc surreprésentées dans cette catégorie. À l'inverse, parmi les personnes ayant répondu se soucier " souvent » de leur prise de poids (510 personnes) on note $34 \%$ de femmes, $54 \%$ d'hommes (alors qu'ils et elles représentent respectivement $44 \%$ de la population de l'enquête) et $12 \%$ de personnes non binaires, queer ou trans. C'est donc du côté des hommes, et notamment des hommes gays, que la proportion de personnes se souciant régulièrement de leur poids est la plus visible. Parallèlement à cela, sur 590 personnes LGBTIQ déclarant ne jamais avoir suivi de régime, nous trouvons $45 \%$ de femmes, $42 \%$ d'hommes et $13 \%$ de personnes trans, queer, ou non binaires. En déplaçant notre regard sur les minorités sexuelles, on observe que le régime n'est donc pas l'apanage que des femmes. À l'inverse, sur 130 personnes ayant suivi un régime (soit $18 \%$ des répondant.e.s), se trouvent $53 \%$ d'hommes, $38 \%$ de femmes et $9 \%$ de personnes non binaires, trans ou queer. Là encore, à bien lire les chiffres de notre enquête, c'est du côté des hommes gays que le différentiel se creuse, venant souligner une fois de plus que le rapport aux corps des hommes gays, des femmes lesbiennes et des personnes trans ou queers n'est certes pas le même, mais, plus encore, que le biais hétérocentré des analyses relatives au rapport aux corps doit être contrebalancé. Bien que nous ne puissions faire abstraction de la pression sociale qui s'exerce sur le corps des femmes tant du point de vue des médias, de la santé, du travail ou encore de la famille [15], le pas de côté que nous effectuons montre que les questions de poids ou de rapport au corps ne sont plus un souci exclusivement féminin. Soulignons également que les femmes font, en moyenne, toutes catégories sociales confondues, plus de régimes que les hommes. Selon Saint-Pol, en 2003 [16], elles étaient $8 \%$ à en avoir fait un alors que seulement $3 \%$ des hommes déclaraient avoir suivi un régime la même année. Cet aparté souligne, concernant les personnes homosexuelles, qu'il ne faut pas conclure hâtivement à une "inversion " des codes et des normes hétérosexuelles. Autrement dit, nous ne postulons pas ici que les hommes gays seraient « comme les femmes hétérosexuelles» dans leur rapport au corps. En réalité, il semble plutôt s'exercer là un rapport aux corps et aux esthétiques, propre aux 
cultures gays et lesbiennes. Pour le dire encore plus nettement, les gays semblent plus soucieux d'un maintien du corps du côté des muscles ou de la minceur que les lesbiennes. Ainsi, le verbatim du questionnaire fait apparaître de plus nombreuses fois les termes de "sport » et de « muscles » ou de « régime » dans les réponses fournies par les hommes gays, et ce peu importe les catégories socio- professionnelles des répondants ou même l'âge de ces derniers (si ce n'est après 65 ans). Néanmoins, cette première conclusion ne saurait non plus pleinement nous satisfaire tant il existe des sous-communautés LGBTIQ qui vivent, à leur manière, leur corporéité. Les critères de beauté et les esthétiques des corps n'épousent pas les mêmes frontières selon les sous-catégories identitaires homosexuelles ou trans. Nous faisons ainsi l'hypothèse que le gras est ainsi très différemment appréhendé selon les communautés sexuelles LGBTI. Les communautés bears, par exemple mettent en avant le ventre et les poils comme critères d'inclusion [17], et cette enquête générale aura notamment eu pour limite de lisser ces sous-cultures esthétiques et corporelles. Toutefois, cette lecture du rapport au corps par les indices identitaires peine à voir le jour en France. À ce stade, cette seconde conclusion reste au stade d'hypothèse, même si une analyse sémiologique des présentations de soi et des photos de profil des applications de rencontre gays témoigne en effet d’une pluralité des esthétiques corporelles [18].

\section{Discussion}

Alors que les travaux francophones manquent cruellement, à l'étranger, certaines publications nous permettent de discuter ces premiers résultats. Ainsi, la revue Psychology of men and masculinity publie en 2016 les résultats d'une étude [19] dont la cible reste les hommes homosexuels. Les auteurs montrent que les hommes gays avaient une image de leurs corps plus dégradée que celle des hommes hétérosexuels- ou se déclarant comme tels (21\% d'image positive de soi chez les hommes gays contre $29 \%$ chez les hommes hétérosexuels) et une insatisfaction notable liée à leur musculature (45\% d'insatisfaction chez les hommes gays contre $30 \%$ chez les hétérosexuels). Dans le même temps, ils se sentent pourtant légèrement moins en surpoids que les hommes hétérosexuels (39\% contre $44 \%)$. Le différentiel d'insatisfaction apparaît donc d'autant plus fort. Ils sont aussi plus nombreux à établir un lien entre leurs précédentes réponses et le rôle des médias. En réponse à l'affirmation « Je me sens obligé d'avoir un corps plus attirant à cause des magazines et de la télévision », $58 \%$ des hommes homosexuels se sont montrés d'accord, contre seulement $29 \%$ des hommes hétérosexuels. À bien lire ces résultats, nous en concluons aux côtés des auteurs de l'étude que les hommes homosexuels sont plus affectés par le jugement des autres. Ceci tend à expliquer également les résultats de notre enquête. 
Toutefois, si les hommes homosexuels et hétérosexuels ressentent la même pression de la part de leurs partenaires, l'insatisfaction corporelle des hommes homosexuels semble avoir des incidences plus importantes sur leurs modifications corporelles par le sport ou la chirurgie notamment. Ainsi, toujours selon Frederick et Assayli [19], les hommes homosexuels sont plus susceptibles que les hommes hétérosexuels de modifier leurs corps. Par exemple, $7 \%$ des hommes homosexuels ont déclaré avoir subi une chirurgie esthétique au cours de la dernière année, contre seulement $1 \%$ des hommes hétérosexuels. De plus, $37 \%$ des hommes homosexuels ont suivi un régime de perte de poids et $12 \%$ ont eu recours à des pilules pour maigrir au cours des 12 derniers mois (pour les hommes hétérosexuels, les chiffres étaient respectivement de $29 \%$ et $5 \%$ ).

Du côté des femmes lesbiennes, certaines études tendent àprouver qu'un IMC élevé est moins sévèrement jugé dans les critères esthétiques lesbiens que gays. Par exemple, Swami et Tovée développent une recherche qualitative auprès de 141 femmes [20]. Ils examinent les prédicteurs de l'attractivité physique féminine en comparant des femmes homosexuelles et hétérosexuelles, se déclarant féministes et non féministes. Les résultats ont montré que, indépendamment de l'orientation sexuelle et de l'engagement féministe de la part des femmes, I'IMC était un prédicteur dominant de l'attrait physique chez les personnes lesbiennes. De plus, les résultats ont également montré que les lesbiennes préfèrent les images de femmes avec un IMC significativement plus élevé que les femmes hétérosexuelles. L'auto-identification en tant que féministe ne semble pas être associée à une préférence pour des IMC plus grands. Les résultats sont discutés en termes de théorie socioculturelle, ce qui suggère que différentes communautés peuvent avoir différents idéaux de beauté, non seulement eu égard à leurs orientations sexuelles mais aussi à leurs engagements concomitants. En parallèle, les études de Strong [21] ont démontré que les hommes et les femmes homosexuel.le.s présentent un risque plus élevé de développer un trouble de l'alimentation comme l'anorexie- et la boulimie. Les auteurs portent leurs regards sur les symptômes liés aux troubles de l'alimentation. Seul $1 \%$ des hommes hétérosexuels remplissaient les critères, contre $10 \%$ des hommes homosexuels. En revanche, $13 \%$ des femmes hétérosexuelles et $9 \%$ des lesbiennes étaient classées comme ayant un trouble de l'alimentation [21].

\section{Conclusion}

À travers l'ensemble de ces chiffres, qu'il convient d'appréhender- comme une première tentative de mesure du rapport au corps comparé entre homosexuel.le.s et 
hétérosexuel-.le.Is, il apparaît aujourd'hui important d'inoculer- plus encore ces recherches communautaires dans la recherche française afin d'établir les parallèles et les différences que les contextes internationaux donnent à voir. Par ailleurs, les questions de poids et les représentations corporelles, marquées par l'effet de la socialisation différenciée entre les sexes, sont également saisies par les appartenances communautaires multiples des individus, notamment celles de type sexuelles ou genrées. II en résulte une nécessaire discussion en matière de prévention et de politique publique à destination des sous-groupes cibles dont on a pu montrer qu'ils n'adoptent pas les mêmes comportements corporels que la population hétérosexuelle. Si les premières données nationales rejoignent celles d'autres études internationales, les méthodologies employées, majoritairement quantitatives, indiquent qu'il faudra dorénavant mener des enquêtes qualitatives, notamment pour déconstruire certaines notions : que veut dire par exemple «suivre un régime » selon les différents sous-groupes de population? À quoi renvoient les insatisfactions corporelles chez les femmes et les hommes issu.e.s de groupes minoritaires?

Références

1. Jedrzejewki T. Enquête Gays et Lesbiennes - Médecine générale (EGaLe-MG) : État des lieux des difficultés rencontrées par les homo-sexuels face à leurs spécificités de santé en médecine générale en France [Thèse de médecine générale]. Paris : Université Paris-Diderot - Paris 7; 18/10/2016. [Visité le 19/08/2018]. En ligne: http://www.bichatlarib.com/publications.documents/5210_JEDRZEJEWSKI_these.pdf.

2. Cassidy F. L'intimité chez les hommes et les femmes homosexuels âgés. Gérontologie et société. 2007;30(3):233-45.

3. Dagorn J, Alessandrin A. La santé des LGBTI à l'école. L'école des parents. 2018;627(2):28-9. Lire aussi Espineira K, Alessandrin A, Thomas MY, Tableau noir: les transidentités et l'école, Cahiers de la transidentité, vol.4, 2014.

4. Richard G. The pedagogical practices of Québec high school teachers relative to sexual diversity. Journal of LGBT Youth. 2015;12(2):113-43. 
5. Amadieu JF. Le poids des apparences : Beauté, amour et gloire. Paris : Odile Jacob ; 2002.

6. Le Talec JY, Jablonsky O, Siberis G. Santé gay. Paris : Pepper ; 2010.

7. Genon C, Chartrain C, Delebarre C. Pour une promotion de la santé lesbienne : état des lieux des recherches, enjeux et propositions. Genre, sexualité \& société. 2009;1. [Visité le 19/08/2018]. En ligne : http://journals.openedition.org/gss/951.

8. de Saint Pol T. Comment mesurer la corpulence et le poids idéal ? Histoire, intérêts et limites de l'indice de masse corporelle. Paris : OSC ; 2007 (collection « Notes \& Documents »).

9. Toulze M. Représentations de l'obésité et corps de l'obèse. In: Meidani A, Alessandrin A (dir.). Parcours de santé Parcours de genre. Toulouse : PUM ; 2018. p. 151-166.

10. Gay L. Homosexus mediaticus. Les cahiers de la transidentité. 2013;2(2):101-16.

11. Institut Roche de l'obésité, Institut national de la santé et de la recherche médicale (Inserm), Kantar Health. ObÉpi 2012 : Enquête épidémiologique nationale sur le surpoids et l'obésité. Neuilly-sur-Seine : Institut Roche de l'obésité ; 2012.

12. Troude-Chastenet P. Surpoids et course à la minceur. Études. 2015;10:29-41.

13. Poulain JP. Sociologie de l'obésité. Paris : PUF ; 2009.

14. Meidani A, Alessandrin A. Cancers et transidentités: une nouvelle population à risques ? Sciences sociales et santé. 2017;35(1): 41-64. Lire aussi : Alessandrin A. Sociologie des transidentités, Cavalier Bleu, 2018.

15. Meidani A. La fabrique du corps. Toulouse : PUM ; 2007.

16. de Saint Pol T. L'obésité en France : les écarts entre catégories sociales s'accroissent. Insee Première. 2007;1123.

17. De Busscher PO. Le monde des bars gays parisiens: différenciation, socialisation et masculinité. Journal des anthropologues. 2000;82- 83:235-49. [Visité le 19/08/2018]. En ligne : https:// journals.openedition.org/jda/3372. 
18. Lemeilleur S. L'expressivité de l'intime sur les dispositifs du web [Thèse de sciences de l'information et de la communication]. Bordeaux : université Bordeaux Montaigne ; 2016.

19. Frederick D, Esayli J. Male body image: The roles of sexual orienta-tion and body mass index across five national U.S. Studies. Psychology of Men \& Masculinity. 2016;17(4):336-51. 20. Swami V, Tovee MJ. The influence of body mass index on the physical attractiveness preferences of feminist and nonfeminist heterosexual women and lesbians. Psychology of women quarterly. 2016;30(3):252-7.

21. Strong S, Williamson D, Netemeyer R, Geer J. Eating disorder symp-toms and concerns about body differ as a function of gender and sexual orientation. Journal of Social and Clinical Psychology. 2000;19(2):240-55. 